NASA Technical Memorandum 105420

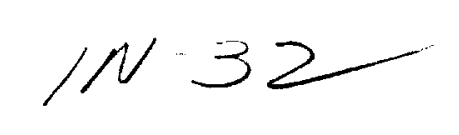

68744

\title{
Advanced Communication Technology Satellite (ACTS) Multibeam Antenna Analysis and Experiment
}

Roberto J. Acosta, Jeffrey M. Larko, and Adabelle Narvaez Lewis Research Center

Cleveland, Ohio

and

Alan R. Lagin

Analex Corporation

Brook Park, Ohio

Prepared for the 1992 AP-S International Symposium

sponsored by the Institute of Electrical and Electronic Engineering Chicago, Illinois, July 18-25, 1992 



\title{
ADVANCED COMMUNICATION TECHNOLOGY SATELLITE (ACTS) MULTIBEAM ANTENNA ANALYSIS AND EXPERIMENT
}

\author{
Roberto J. Acosta, Jeffrey M. Larko and Adabelle Narvaez \\ National Aeronautics and Space Administration \\ Lewis Research Center \\ Cleveland, Ohio 44135 \\ Alan R. Lagin \\ Analex Corporation \\ Brook Park, Ohio 44142
}

\begin{abstract}
One of the most important aspects of a satellite communication system design is the accurate estimation of antenna performance degradation (Pointing error, end coverage gain, peak gain degradation, etc. are of main concern). The thermal or dynamic distortions of a reflector antenna structural system can affect the far-field antenna power distribution in at least four ways. 1. The antenna gain is reduce, 2. The main lobe of the antenna can be mispointed thus shifting the destination of the delivered power away from the desired locations 3 . The main lobe of the antenna pattern can be broadened thus spreading the RF power over a larger area than desired 4 . The antenna pattern sidelobes can increase thus increasing the chances of interference among adjacent beams of a multiple beam antenna system or with antenna beams of other satellites. The in-house developed NASA Lewis Research Center thermal/structural/RF analysis program was designed to accurate simulate the ACTS in-orbit thermal environment and predict the RF antenna performance. The program combines well establish computer programs (TRASYS, SINDA and NASTRAN) with a dual reflector-physical optics RF analysis program. The ACTS multibeam antenna configuration is analyzed and several thermal cases are presented and compared with measurements (pre-flight).
\end{abstract}

\section{Introduction}

The ACTS multibeam antenna system (figure 1.) consists of a separate Ka-band $(30 / 20 \mathrm{GHz})$ antennas, each with horizontal and vertical polarization subreflectors. The offset Cassegrian antenna system provides two hopping spot beam families (east and west) plus three fixed spot beams. The east and west families are discriminated by polarization. The east family of beams comprises an east scan sector and additional spot beams for isolated-location coverage outside the sector. The west family comprises a west scan sector, additional spot beams for isolated-location coverage outside the sector, and a steerable spot beam. Within the beams access will be by demand-assigned multiple access (DAMA) using time division multiple access (TDMA) transmission. The multibeam communication center package provides the means for receiving, processing, switching, amplifying, and transmitting signals that carry high-speed digital communication traffic. 


\section{ACTS Multibeam Antenna Configuration (transmitting case)}

The ACTS dual reflector (front sub-reflector) geometry (figure 2) is analyzed. This configuration consists of parabolic main reflector with a focal length of 132 inches, and hyperbolic sub-reflector (cassegrian configuration). The feed is located at the Cleveland spot beam. The feed is modeled with measured $E$ and $H$ plane far-field antenna pattern.

\section{Discussion and Results.}

The ACTS MBA transmitting configuration was simulated using the thermal/structure/dual reflector-physical optics computer program. Several cases are presented and its antenna performance calculated. One of the limitation of our model is that it does not include the grided characteristics of the front sub-reflector. It assumes that the sub-reflector is a solid perfect conductor. Also the points generated by thermal/structure portion of the analysis program are interpolated by using either polynomial spline algorithm (3th order) or a 4 th order polynomial. The ideal far-field antenna performance for the Cleveland spot beam (case 1) is shown in figure 3 . The surface points were generated using analytic equations. Its calculated peak directivity is 55.45 $\mathrm{db}$. Figure 4 shows the simulated pre-flight (case 2) antenna far-field pattern. In this case the surface points are measured with a main reflector rms of 5 mils. The calculated directivity was $54.45 \mathrm{db}$. Case 3 simulates an in-orbit case representing the spacecraft max cold thermal cycle. The antenna performance is presented in figure 5. The calculated directivity is $54.01 \mathrm{db}$. For comparison purposes the measured pre-flight antenna pattern for the Cleveland spot beam is presented in figure 6 .

All the antenna pattern are very similar is shape to the measured pattern, slight differences in gain (less $0.5 \mathrm{db}$ ) and sidelobe level (1-3db) are observed. The discrepancies are mainly due to sub-reflector modeling, feed modeling and surface errors interpolation. The overall simulation results were in agreement with the measurements. The thermal/structure/RF analysis program could provide an indirect means of verifying in-orbit antenna performance.

\section{References}

1. Acosta, R.J.;Active Feed Array Compensation for Reflector Antenna Surface Distortions, NASA TM-100826, June 1988.

2. Sharp, G.R.;Acosta R.J.;Bobisnsky, E.A. and Shaker, F.J.; A Conceptual System Design for Antenna Thermal and Dynamic Distortion Compensation Using Phased Array Feed. Second NASA/DOD Control/Structures Interaction Technology Conference, Colorado Spring, Co, Novemeber 17-19, 1987. 


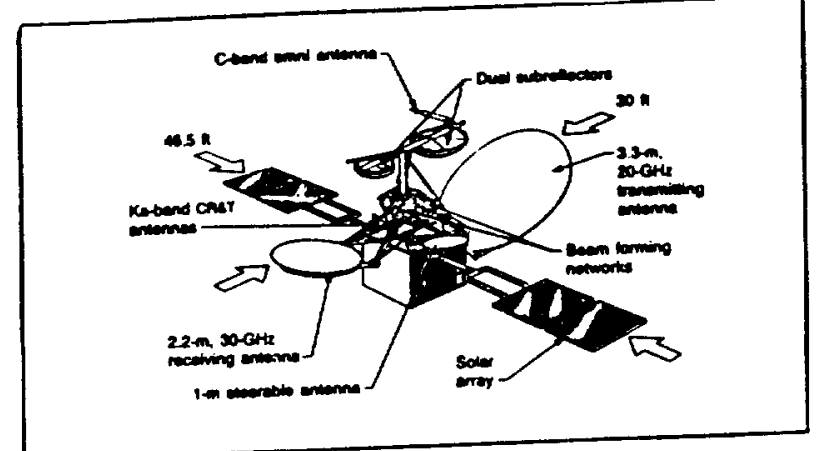

Figure 1, The ACTS mult ibean antenna system
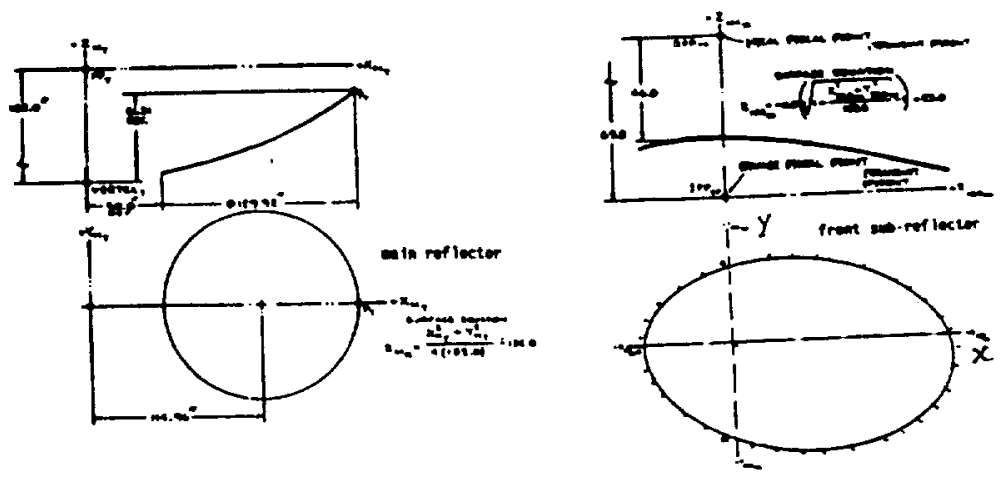

f1em 2. Mers an tronselttin ref lecter montry

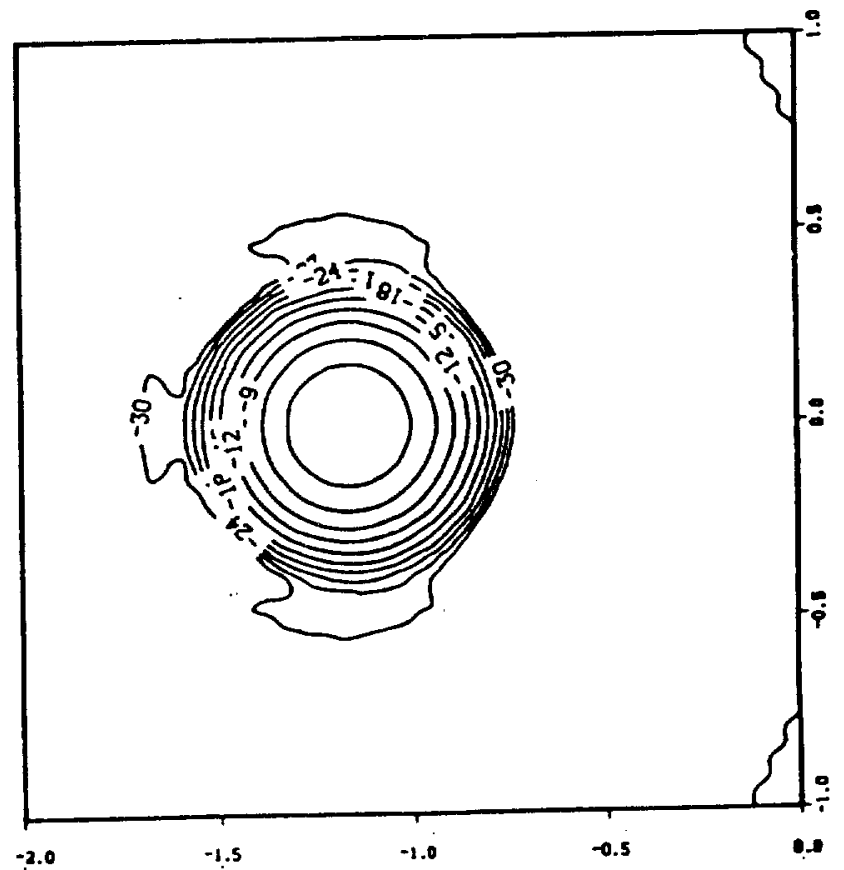

Figure 3, Simulated ideal case far-fteld antenna pattern

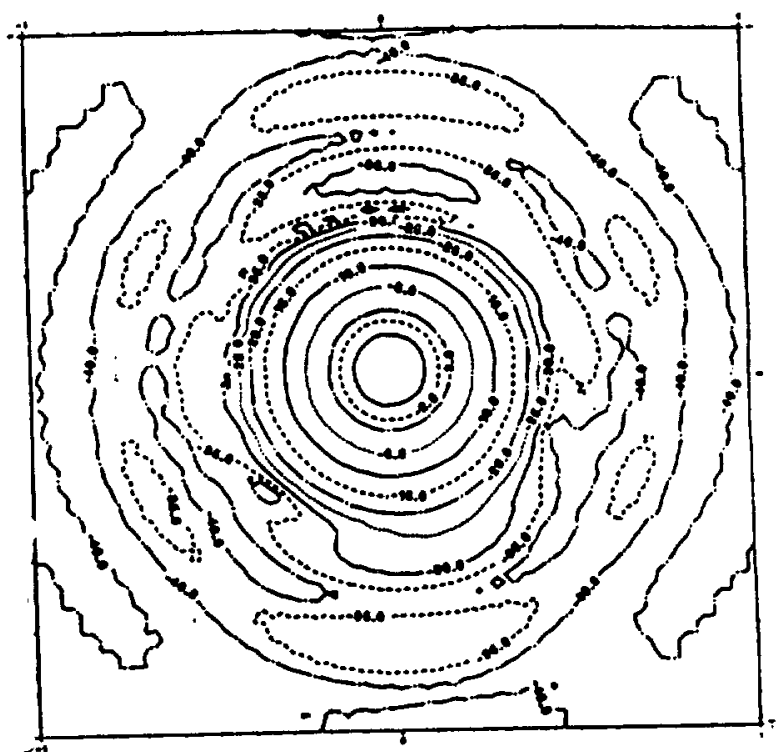

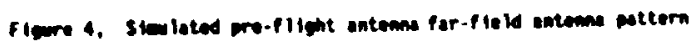

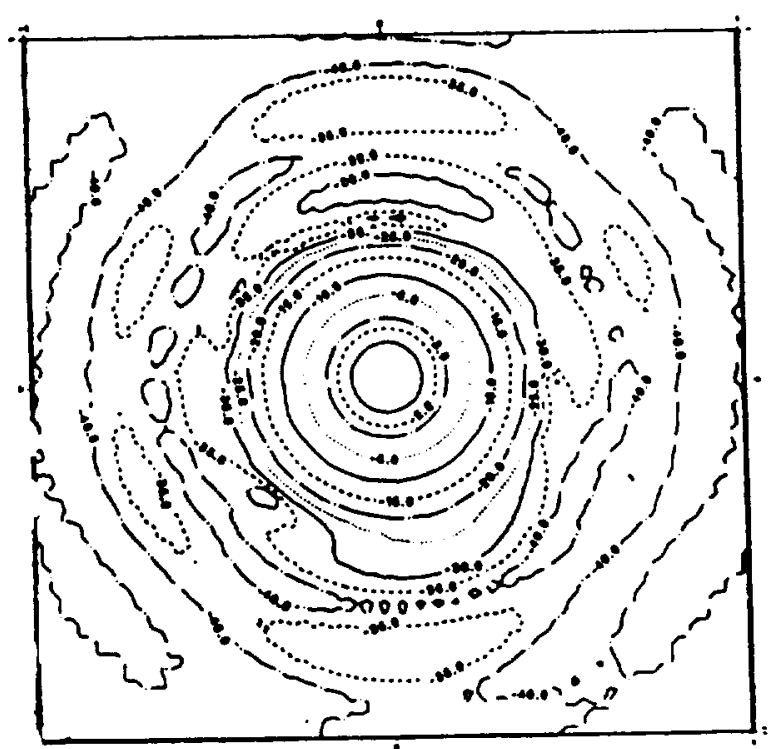

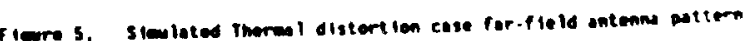

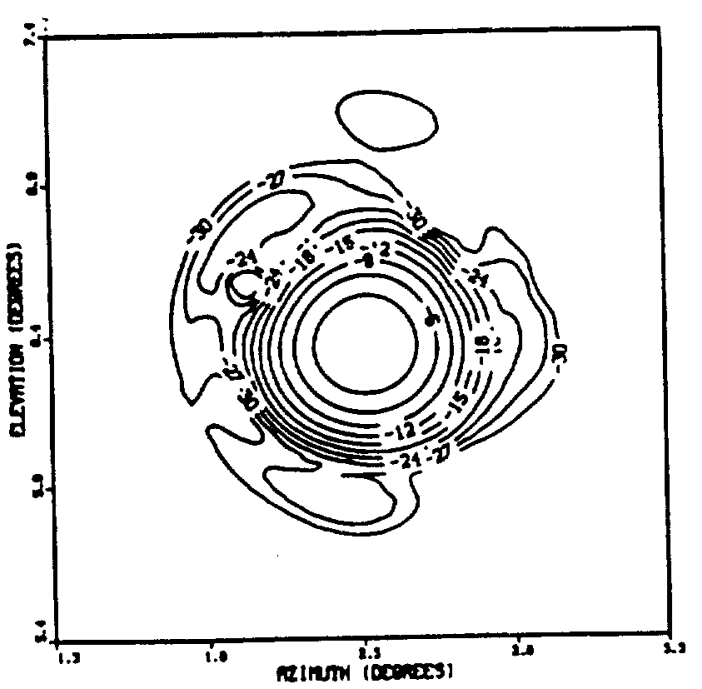

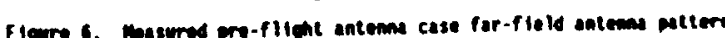

ORIGHAL PAGE IS OF POOR QUALITY 


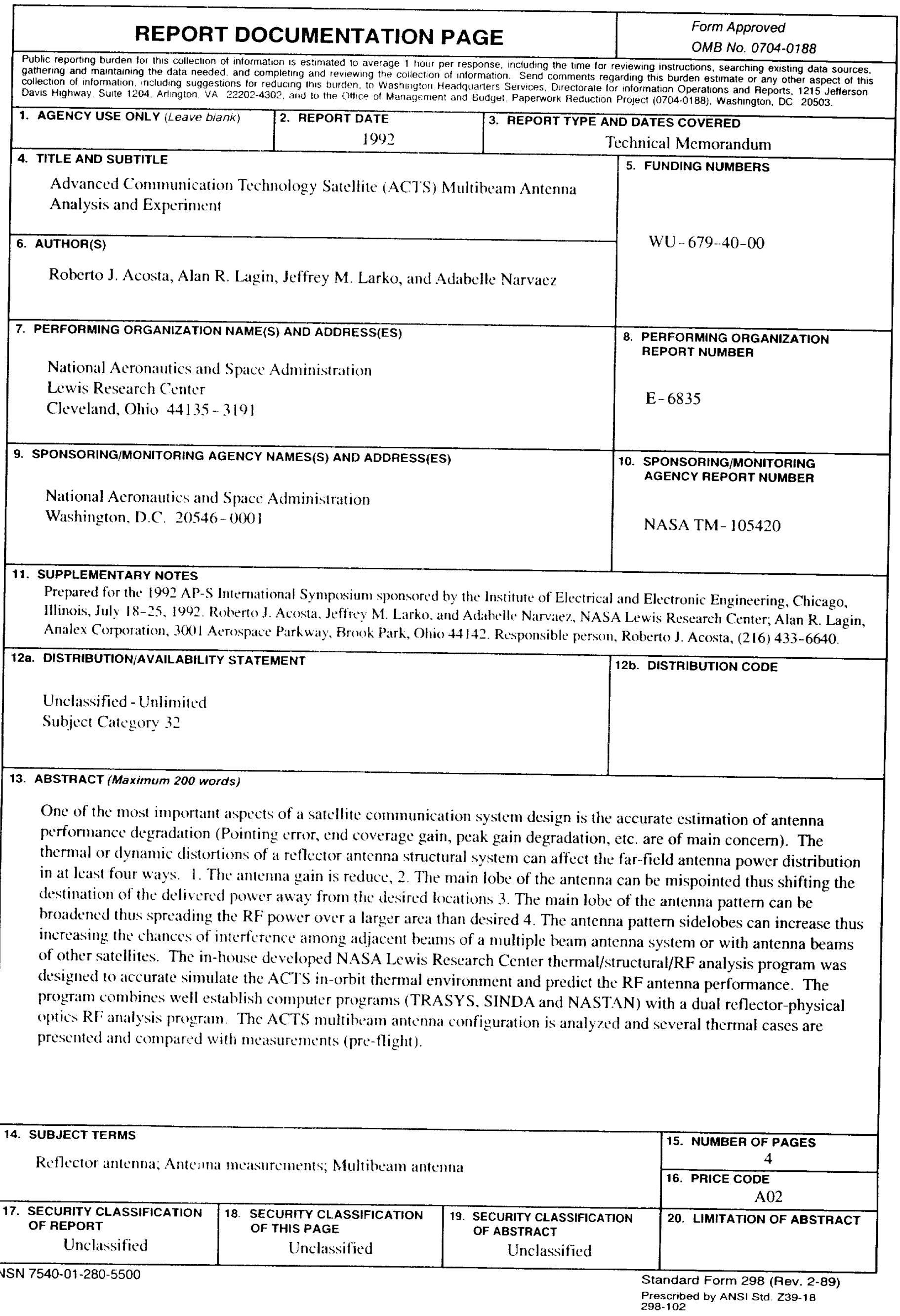


National Aeronautics and

Space Administration

Lewis Research Center

Cleveland, Ohio 44135

Otitatal Duainease

Penetry for Privet Ues $\$ 300$

\section{ADDRESS CORRECTION REQUESTED}

Postage and Fees Pard National Aeronautics and Space Administration

NASA 451 